\title{
Indurated Mass on the Right Central Back
}

John Hassani, DO; Paul Chu, MD; Adriana Ros, DO

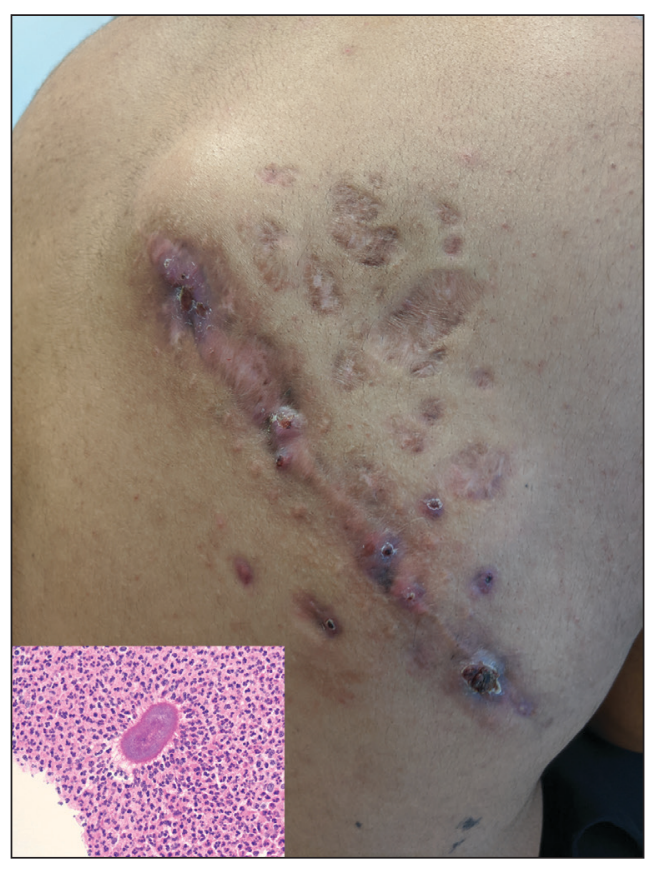

A 26-year-old Guatemalan man who was a former carpenter presented with an indurated, nontender, nonpruritic, subcutaneous mass on the right central back with multiple draining sinus tracts on the surface and several depressed circular atrophic scars on the periphery of the mass. He noticed that the lesion began as a pustule 1.5 years prior and gradually enlarged. He denied any trauma, insect bites, fever, chills, headaches, weight loss, or travel history (he relocated to the United States 3.5 years ago) prior to the skin eruption. A biopsy was performed by an outside dermatologist 1 year prior to the current presentation, with a diagnosis of Pityrosporum folliculitis. Throughout his clinical course, treatment with oral antifungals, oral doxycycline, and topical clindamycin all failed. The mass was removed by plastic surgery 1 year prior.

A tissue biopsy for histology and culture was obtained at presentation to our institution. Laboratory findings showed that the basic metabolic panel was within reference range. Chest radiography indicated no active disease. A tuberculosis screening was negative. A bacterial culture of the lesion identified no growth after 48 hours. Our tissue biopsy revealed fibrosing granulation tissue, but the surgical pathology from a prior mass excision revealed sinus tracts with suppuration, evidence of scarring, foreign body giant cell reaction, and a characteristic finding (inset: H\&E, original magnification ×200).

\section{WHAT'S YOUR DIAGNOSIS?}
a. actinomycetoma
b. botryomycosis
c. cutaneous tuberculosis
d. foreign body reaction
e. sarcoma

PLEASE TURN TO PAGE E30 FOR THE DIAGNOSIS 


\section{THE DIAGNOSIS: Actinomycetoma}

$\square$ istopathology revealed evidence of an actinomycete organism within the suppuration, consistent with actinomycosis (quiz image [inset]). Given the clinical presentation and histopathologic findings, our patient was diagnosed with actinomycetoma.

Actinomycetoma is an indolent, progressive, subcutaneous infection characterized by a well-known clinical triad of tumefaction/subcutaneous mass, draining sinuses, and an exudate containing grains on microscopy. The sinus tracts are formed from the chronic infectious process that destroys tissue, creating tunnels. This infectious disease of soft tissue is a clinical subset of mycetoma, which is categorized as eumycetoma (fungal) and actinomycetoma (bacterial). Actinomycetoma resembles the behavior of insidious and chronic fungal infections; however, most mycetoma infections are bacterial. ${ }^{1,2}$ Actinomycetoma may be confused with actinomycosis, which is caused by Actinomycoses species, commensal organisms commonly located on the teeth and oral mucosa in association with other microorganisms that may pathogenically cause cervicofacial actinomycosis. ${ }^{3,4}$ Actinomycetoma can be caused by Nocardia, Streptomyces, and Actinomadura. ${ }^{2,5}$ The foot is the most common location of involvement followed by the thoracic region. It is more common in tropical or equatorial locations and may be contracted through exposure to soil or wood. ${ }^{5}$ Mycetoma is considered a neglected tropical disease by the World Health Organization. ${ }^{1}$ In tropical countries, this disease may go undiagnosed or untreated for so long that surgical amputation may be the only effective treatment.

Actinomycetoma commonly is identifiable by direct microscopy, Gram stain, or bacterial culture, with Gram stain being more sensitive than bacterial culture. ${ }^{3}$ It is important to indicate the suspected organism to the microbiology laboratory because common bacterial pathogens are detected within 24 to 48 hours, but the causative microorganism in actinomycetoma may require up to 4 weeks for culture, ${ }^{2}$ leading to possible false negatives due to inadequate culture time. ${ }^{3}$ Histopathology of actinomycotic infections will demonstrate granulomatous inflammation, focal suppuration, and the presence of grains (ie, a colony of filamentous bacteria in a stellate shaped mass)(quiz image [inset]).

The gold standard of treatment is trimethoprimsulfamethoxazole for up to several years. ${ }^{4,5}$ Amoxicillinclavulanic acid, dapsone, amikacin, streptomycin, and beta-lactams have been used successfully., ${ }^{2,5}$ The treatment course is dependent on clinical severity and location of the disease. The cure rate with appropriate antibiotics can be as high as $90 \%, 2,5$ and thus surgical intervention can be avoided.

In the differential, cutaneous tuberculosis would show tuberculoid granulomas with epithelioid histiocytes with possible caseation on histopathology, typically alongside positive tuberculosis screening. Botryomycosis has a similar clinical presentation of a swollen or indurated lesion with draining sinus tracts, but it less commonly occurs on the trunk. Histopathology also is a close mimic of actinomycetoma with a small grain inside a suppurative infiltrate; however, it has no filamentous bacteria. A foreign body reaction would not histologically present with suppuration or grains, and draining sinuses typically would not be seen on clinical presentation. Sarcoma is a neoplastic process and most commonly would show a proliferation of cells with soft tissue or bone origin on histopathology and not primarily an inflammatory cell process. ${ }^{6}$

\section{REFERENCES}

1. Verma P, Jha A. Mycetoma: reviewing a neglected disease. Clin Exp Dermatol. 2019;44:123-129.

2. Valour F, Sénéchal A, Dupieux C, et al. Actinomycosis: etiology, clinical features, diagnosis, treatment, and management. Infect Drug Resist. 2014;7:183-197.

3. Bennhoff DF. Actinomycosis: diagnostic and therapeutic considerations and a review of 32 cases. Laryngoscope. 1984;94:1198-1217.

4. Welsh O, Vera-Cabrera L, Welsh E, et al. Actinomycetoma and advances in its treatment. Clin Dermatol. 2012;30:372-381.

5. Arenas R, Fernandez Martinez RF, Torres-Guerrero E, et al. Actinomycetoma: an update on diagnosis and treatment. Cutis. 2017;99:E11-E15.

6. Weedon D. Weedon's Skin Pathology. 3rd ed. Churchill Livingstone Elsevier; 2010. 